Publisher: Research Council of Science and Technology, Biratnagar, Nepal p.20

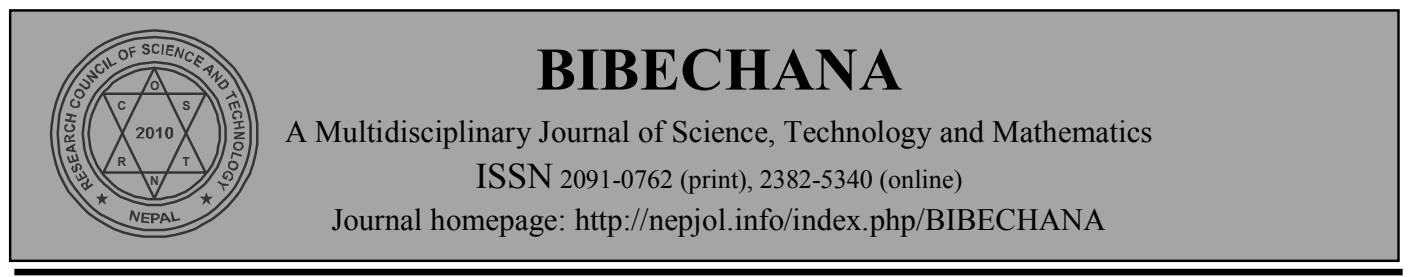

\title{
Thermodynamic and structural behaviour of Tl-Na liquid alloy
}

\author{
S. K. Yadav ${ }^{1^{*}}$, L.N. Jha ${ }^{2}$, D. Adhikari ${ }^{\text {*** }}$ \\ ${ }^{1}$ Department of Physics, Mahendra Morang Adarsh Multiple Campus (Tribhuvan University) \\ Biratnagar, Nepal \\ ${ }^{2}$ Central Department of Physics, Tribhuvan University, Kirtipur, Kathmandu, Nepal \\ ${ }^{* *}$ E-mail: adksbdev@yahoo.com \\ "E-mail: yadavshashit@yahoo.com \\ Accepted for publication: December 8, 2014
}

\begin{abstract}
In this work we intend to study the thermodynamic and microscopic structural properties of $\mathrm{Tl}-\mathrm{Na}$ liquid alloy at $673 \mathrm{~K}$ on the basis of regular associated solution model. We have estimated the mole fractions of the complex and the free monomers assuming the complex TlNa as energetically favoured in the initial melt. In thermodynamic properties, we have computed free energy of mixing $\left(G_{M}\right)$, enthalpy of mixing $\left(H_{M}\right)$, entropy of mixing $\left(S_{M}\right)$ and activities of the monomers $\left(a_{T l}\right.$ and $\left.a_{N a}\right)$. The compositional contributions of the heat of mixing and the heat of formation of the complex to the net enthalpy change have also been studied. The microscopic structural properties of the alloy have been studied by computing concentration fluctuation in the long wavelength limit $\left(S_{C C}(0)\right)$, chemical short range order parameter $\left(\alpha_{1}\right)$ and the ratio of mutual to intrinsic diffusion coefficients $\left(D_{M} / D_{i d}\right)$. Both the theoretical and the experimental values of $S_{C C}(0)$ are found to be less than the ideal values at all compositions which indicates the ordering nature of Tl-Na liquid alloy at $673 \mathrm{~K}$. All the interaction energy parameters are found to be negative and temperature dependent. The liquid system is found to be moderately interacting.
\end{abstract}

DOI: http://dx.doi.org/10.3126/bibechana.v12i0.11784

(C) 2014 RCOST: All rights reserved.

Keywords: Tl-Na liquid alloy; energetically favoured; interaction energy parameters; temperature dependent; moderately interacting.

\section{Introduction}

The multidimensional uses and the dynamical properties of binary liquid alloys have drawn considerable attentions of the present day researches. Most of the researches are confined to develop new alloys and to investigate their thermodynamic and structural behaviors to meet the greater demands of the today's world. Generally, the alloys are grown from their liquid state and hence the study of thermodynamic and structural properties of the initial melt play important role for their uses in the solid state. Several theoretical models till now have been proposed to study the thermodynamic and structural properties of the liquid alloys [1-12]. There also exist immense interest in developing and devising the Tl-based alloys because of their involvements in the manufacture of electronic components, optical lenses, semiconductor materials, gamma radiation detectors, jewelry, green fireworks and wear and tear resistant shafts [13-16]. In this work, we intend to study and predict the 
thermodynamic and microscopic structural properties of Tl-Na liquid alloy at $673 \mathrm{~K}$ on the basis of regular associated solution model [17-22].

The regular associated solution model is based on the assumption of the presence of the complexes in the liquid state. When the atoms of types $\mathrm{A}$ and $\mathrm{B}$ are mixed in the liquid state, there are associations among A, B; A, A and B, B called 'complexes', 'pseudomolecules', 'clusters' or simply 'associations' [6]. The expressions for the different thermodynamic and structural function are derived on the basis of the existence of unequal interactions among complex and the unassociated atoms. Prigogine and Defay [23] proposed that the interaction energies and the concentrations of the two free monomers and the complex would determine the ternary activity coefficients. Jordan [21] further elaborated that the activities of the free monomers and the complexes can be estimated by considering the liquid solution as the ternary system and attributing the deviations from the ideal behaviour to the shortrange neighborhood of interactions. He named the solution as regular associated solution and applied this concept in the congruently melting semiconductors $\mathrm{Zn}-\mathrm{Te}$ and $\mathrm{Cd}-\mathrm{Te}$. This concept was employed by Osamura and Predel [24] to the In-Sb system but derived the necessary equations by neglecting the higher order terms. Later, Lele and Ramchandrarao [19] further extended this model and verified its utility for the determination of the thermodynamic properties and the activities for $\mathrm{Mg}-\mathrm{Sn}$ and In-Sb liquid alloys.

The phase diagram of the Tl-Na alloys, [25] shows the existence of two phases, such as $\delta$, "TlNa" (with fcc protopype structure and $\gamma, " \mathrm{Na}_{2} \mathrm{Tl}$ " (with end centered-orthorhombic structure). In this work we have assumed the presence of TlNa complex as energetically favoured in the liquid state of Tl-Na alloy at $673 \mathrm{~K}$ and studied its thermodynamic and microscopic structural properties.

The expressions for the various thermodynamic and structural functions are presented in the section 2, the results and discussion are presented in section 3 and the conclusions of this work are presented in the section 4 .

\section{Theory}

When the atoms of the type $\mathrm{A}(=\mathrm{Tl})$ and $\mathrm{B}(=\mathrm{Na})$ are mixed at a temperature near to their melting point, there is formation of the complex of the form $\mathrm{AB}$ (TlNa). Let one mole of the binary liquid contain $n_{1}$ atoms of monomer $\mathrm{A}(=\mathrm{In})$ and $n_{2}$ atoms of monomer $\mathrm{B}(=\mathrm{Bi})$. Following Lele and Ramchandrarao [19], the chemical complexes of the form $A_{\mu} B\left(A_{\mu} B \Leftrightarrow \mu A+B\right)$ is assumed to exists in the initial melt, where $\mu$ is a small integer whose value is determined from the compound forming concentration $(=\mu /(\mu+1))$ in the solid state. The liquid mixture is thus the ternary mixtures of unassociated atoms $\mathrm{A}(=\mathrm{Tl})$ and $\mathrm{B}(=\mathrm{Na})$ and the complex $A_{\mu} B(=T l N a)$, where $\mu=1$. If $n_{A}, n_{B}$ and $n_{A_{\mu} B}$ moles are the concentrations of $\mathrm{A}, \mathrm{B}$ and $A_{\mu} B$ in the melt then according to the conservation of mass, in the partially associated solution the formation of the complex $A_{\mu} B$ requires $n_{1}=n_{A}+$ $\mu n_{A_{\mu} B}$ and $n_{2}=n_{B}+n_{A_{\mu} B}$. But the thermodynamic and microscopic structural behaviours of the complexes in the melt are governed by their true mole fractions $x_{A}, x_{B}$ and $x_{A_{\mu} B}$ in spite of their gross mole fractions $x_{1}$ and $x_{2}$, where $x_{1}=n_{1} / n_{1}+n_{2}, x_{2}=n_{2} / n_{1}+n_{2}, x_{A}=n_{A} / n_{A}+n_{B}+n_{A_{\mu} B}$ , $x_{B}=n_{B} / n_{A}+n_{B}+n_{A_{\mu} B}$ and $x_{A_{\mu} B}=n_{A_{\mu} B} / n_{A}+n_{B}+n_{A_{\mu} B}$.

Using above expressions, the equations for the true mole fractions can be derived as

$$
x_{A}=x_{1}-\mu x_{2} x_{A_{\mu} B} \quad \text { and } x_{B}=x_{2}-\left(1-\mu x_{2}\right) x_{A_{\mu} B}
$$


Following Prigogine and Defay [23], in the regular associated liquid solution the gross chemical potentials of the components 1 and 2 are equal to the chemical potentials of the free monomers $\mathrm{A}$ and B. According to Jordan, [21] the activity coefficients of monomers $\gamma_{A}, \gamma_{B}$ and complex $\gamma_{A_{\mu} B}$ can be represented in terms of pairwise interaction energies as

$$
\begin{aligned}
& \mathrm{RT} \ln \gamma_{A}=x_{B}^{2} \omega_{12}+x_{A_{\mu} B}^{2} \omega_{13}+x_{B} x_{A_{\mu} B}\left(\omega_{12}-\omega_{23}+\omega_{13}\right) \\
& \text { RT } \ln \gamma_{B}=x_{A_{\mu} B}^{2} \omega_{23}+x_{A}^{2} \omega_{12}+x_{A} x_{A_{\mu} B}\left(\omega_{23}-\omega_{13}+\omega_{12}\right) \\
& \text { RT } \ln \gamma_{A_{\mu} B}=x_{A}^{2} \omega_{13}+x_{B}^{2} \omega_{23}+x_{A} x_{B}\left(\omega_{13}-\omega_{12}+\omega_{23}\right)
\end{aligned}
$$

where $\omega_{12}, \omega_{13}$ and $\omega_{23}$ represents the pairwise interaction energies for the species A, B; A, $A_{\mu} B$ and $\mathrm{B}, A_{\mu} B$ respectively. $\mathrm{T}$ and $\mathrm{R}$ represent temperature and universal real gas constant respectively.Following Lele and Ramchandrarao,[19] the equilibrium constant for the reaction $A_{\mu} B \Leftrightarrow \mu A+B$ can given by following equation

$$
k=\frac{x_{A}^{\mu} x_{B} \gamma_{A}^{\mu} \gamma_{B}}{x_{A_{\mu} B} \gamma_{A_{\mu} B}}
$$

Solving Equations (2) and (3), the relation for the equilibrium constant can be obtained as

$$
\begin{aligned}
& \operatorname{lnk}=\ln \left(\frac{x_{A}^{\mu} x_{B}}{x_{A_{\mu} B}}\right)+\frac{\omega_{12}}{R T}+\frac{\omega_{12}}{R T}\left[\mu x_{B}\left(1-x_{A}\right)+x_{A}\right]+\frac{\omega_{13}}{R T}\left[\mu x_{A_{\mu} B}\left(1-x_{A}\right)-x_{A}\right]+ \\
& \frac{\omega_{23}}{R T}\left[x_{A_{\mu} B}\left(1-\mu x_{B}\right)-x_{B}\right]
\end{aligned}
$$

The expression for free energy of mixing $\left(G_{M}\right)$ is given as [19]

$$
\begin{aligned}
& \frac{G_{M}=}{1+\mu x_{A_{\mu} B}} R T\left[\left(x_{A} x_{B} \frac{\omega_{12}}{R T}+x_{A} x_{A_{\mu} B} \frac{\omega_{13}}{R T}+x_{B} x_{A_{\mu} B} \frac{\omega_{23}}{R T}\right)+\left(x_{A} \ln X_{A}+x_{B} \ln X_{B}+x_{A_{\mu} B} \ln X_{A_{\mu} B}\right)+\right. \\
& \left.x_{A_{\mu} B} \operatorname{lnk}\right]
\end{aligned}
$$

The other thermodynamic functions, such as heat of mixing $\left(H_{M}\right)$, entropy of mixing $\left(S_{M}\right)$ and concentration fluctuation in the long wavelength limit $\left(S_{C C}(0)\right)$ are related with free energy of mixing $\left(G_{M}\right)$ by the following standard thermodynamic relations

$$
\begin{aligned}
& H_{M}=G_{M}-\mathrm{T}\left(\frac{\partial G_{M}}{\partial T}\right)_{P} \\
& S_{M}=\frac{H_{M-G_{M}}}{T} \\
& S_{C C}(0)=\operatorname{RT}\left(\frac{\partial^{2} G_{M}}{\partial x_{1}^{2}}\right)_{T, P}^{-1} \\
& S_{C C}(0)=x_{2} \alpha_{1}\left(\frac{\partial \alpha_{1}}{\partial x_{1}}\right)_{T, P}^{-1}=x_{1} \alpha_{2}\left(\frac{\partial \alpha_{2}}{\partial x_{2}}\right)_{T, P}^{-1}
\end{aligned}
$$

Using Equation (5) in Equation (6a), the expression for the enthalpy of mixing can be obtained as

$$
\begin{aligned}
H_{M}=\frac{1}{1+\mu x_{A_{\mu} B}} & {\left[\left(x_{A} x_{B} \frac{\omega_{12}}{R T}+x_{A} x_{A_{\mu} B} \frac{\omega_{13}}{R T}+x_{B} x_{A_{\mu} B} \frac{\omega_{23}}{R T}\right)-\mathrm{T}\left(x_{A} x_{B} \frac{\partial \omega_{12}}{\partial T}+x_{A} x_{A_{\mu} B} \frac{\partial \omega_{13}}{\partial T}+\right.\right.} \\
& \left.\left.x_{B} x_{A_{\mu} B} \frac{\partial \omega_{23}}{\partial T}\right)-x_{A_{\mu} B} R T^{2} \frac{d l n k}{d T}\right]
\end{aligned}
$$

The expression for the concentration fluction in the long-wavelength limit $\left(S_{C C}(0)\right)$ can be obtained by using Equation (5) in Equations (6c) and (6d) as 
$S_{C C}(0)=1 /\left[\left(\frac{1}{1+\mu x_{A_{\mu} B}}\right)\left\{\frac{2}{\mathrm{RT}}\left(x_{A}^{\prime} x_{B}^{\prime} w_{12}+x_{A}^{\prime} x_{A_{\mu} B}^{\prime} w_{13}+x_{B}^{\prime} x_{A_{\mu} B}^{\prime} w_{23}\right)+\left(\frac{x_{A}^{\prime 2}}{x_{A}}+\frac{x_{B}^{\prime 2}}{x_{B}}+\frac{x_{A_{\mu} B}^{\prime 2}}{x_{A_{\mu} B}}\right)\right\}\right]$

Here, $\frac{\partial^{2} G_{M}}{\partial x_{1}^{2}}>0$ for $\frac{\partial G_{M}}{\partial x_{1}}=0$. The prime denotes the differentiations with respect to concentrations, and $x_{A}^{\prime}$ and $x_{B}^{\prime}$ are obtained by using Equation (1). $x_{A_{\mu} B}^{\prime}$ is obtained using Equation (4) by using the condition $\frac{d \operatorname{lnk}}{d x_{1}}=0$. [5,6,17] The factor $\left(1+\mathrm{p} x_{A_{\mu} B}\right)^{-1}$, which appears as a coefficient of all terms containing $x_{A}, x_{B}$ and $x_{A_{\mu} B}$ in Equations (5), (7) and (8), is a result of change in the basis for expressing mole fractions of species $\mathrm{A}, \mathrm{B}$ and $A_{\mu} B$ from that used for $x_{1}$ and $x_{2}$. The concentration fluctuation in long wavelength limit $\left(S_{C C}(0)\right)$ has been developed as an important tool to understand the nature of atomic ordering in the binary liquid alloys. At a given concentration, if $S_{C C}(0)<S_{C C}^{i d}(0)$ then ordering (unlike atoms pairing) is expected and if $S_{C C}(0)>S_{C C}^{i d}(0)$ then segregation (like atoms pairing) is expected, where $S_{C C}^{i d}(0)=x_{1} x_{2}$. The experimental values of $S_{C C}(0)$ can be obtained from Equation (6d).[26,27]

The degree of local ordering in such liquid systems can be studied by estimating Warren-Cowley short range order parameter $\left(\alpha_{1}\right)$ with the knowledge of $\left(S_{C C}(0)\right)$.[12, 27, 28] The expression for $\alpha_{1}$ in terms of $S_{C C}(0)$ is given as

$\alpha_{1}=\frac{S-1}{S(Z-1)+1}$ where, $S=\frac{S_{C C}(0)}{S_{C C}^{i d}(0)}$ and $S_{C C}^{i d}(0)=x_{1} x_{2}$

where $Z$ is the coordination number and $Z=10$ is taken for our calculation. We note that varying the value of $Z$ doesn't have any effect on the position of the minima of $\alpha_{1}$; the effect is to vary the depth while the overall features remains unchanged.

The greater insight of microscopic structural properties can be done by evaluating diffusion coefficient which is expressed in terms of $S_{C C}(0)$ by using Darken thermodynamic equation [29, 30] as

$$
\frac{D_{M}}{D_{i d}}=\frac{x_{1} x_{2}}{S_{C C}(0)}
$$

where, $D_{M}$ stands for the chemical or mutual diffusion coefficient and $D_{i d}$ stands for the intrinsic diffusion coefficient for an ideal mixture which are related as

$$
D_{M}=D_{i d} \frac{\partial \ln a_{A}}{\partial x_{1}}, \text { with } D_{M}=x_{1} D_{B}+x_{2} D_{A}
$$

where, $D_{A}$ and $D_{B}$ are the self-diffusion coefficient of pure components A and B respectively.

The pairwise interaction energy parameters and the equilibrium constant are determined by the following procedures:

According to the regular associated solution model $x_{1} \gamma_{1}=x_{A} \gamma_{A}$ and $x_{2} \gamma_{2}=x_{B} \gamma_{B}$, where $\gamma_{1}$ and $\gamma_{2}$ are the gross activity coefficients of components 1 and 2 respectively. They can thus be related as follows

$\ln \gamma_{1}=\ln \gamma_{A}+\ln \left(\frac{x_{A}}{x_{1}}\right)$

and, $\ln \gamma_{2}=\ln \gamma_{B}+\ln \left(\frac{x_{B}}{x_{2}}\right)$

On solving Equations (2a) and (2b), we obtain

$\frac{\omega_{13}}{R T}=\frac{x_{B} \ln \left(\frac{\alpha_{2}}{x_{B}}\right)+\left(1-x_{B}\right) \ln \left(\frac{\alpha_{1}}{x_{A}}\right)-x_{B}\left(1-x_{B}\right) \frac{\omega_{12}}{R T}}{x_{A_{\mu} B}^{2}}$ 
$\frac{\omega_{23}}{R T}=\frac{x_{A} \ln \left(\frac{\alpha_{1}}{x_{A}}\right)+\left(1-x_{A}\right) \ln \left(\frac{\alpha_{2}}{x_{B}}\right)-x_{A}\left(1-x_{A}\right) \frac{\omega_{12}}{R T}}{x_{A_{\mu} B}^{2}}$

where $\alpha_{1}=x_{1} \gamma_{1}$ and $\alpha_{2}=x_{2} \gamma_{2}$ are activities of components 1 and 2 respectively.

The pairwise interaction energies, the equilibrium constants and the activity coefficients at infinite dilution for $\mu=1$ can be related as [19]

$\ln \gamma_{1}^{0}=\frac{\omega_{12}}{R T}+\left[1-\left(1+k \exp \frac{\omega_{23-\omega_{12}}}{R T}\right)^{-1}\right]$

$\ln \gamma_{2}^{0}=\frac{\omega_{12}}{R T}+\left[1-\left(1+k \exp \frac{\omega_{13-\omega_{12}}}{R T}\right)^{-1}\right]$

where $\gamma_{1}^{0}$ and $\gamma_{2}^{0}$ are the activity coefficients at zero concentrations of the free monomers $\mathrm{A}$ and $\mathrm{B}$ respectively. On solving Equations (4), (11) and (12), we obtain

$$
\begin{aligned}
\ln k+\frac{\omega_{13}}{R T} & =\left(\frac{1+x_{A}}{x_{A_{\mu} B}}\right) \ln \left(\frac{\alpha_{1}}{x_{A}}\right)+\frac{x_{B}}{x_{A_{\mu} B}}\left[\ln \left(\frac{\alpha_{1}}{x_{A}}\right)-\frac{\omega_{12}}{R T}\right]+\ln \left(\frac{a_{1}^{\mu} a_{2}}{x_{A_{\mu} B}}\right) \\
& =\ln \gamma_{1}^{0}-\ln \left[1-\gamma_{2}^{0} \exp \left(-\omega_{12} / R T\right)\right]
\end{aligned}
$$

The expression for the complex at equiatomic composition i.e., at $x_{1}=x_{2}=0.5 ; x_{A}=x_{B}$ can be written as

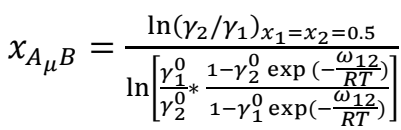

The mole fraction of the complex and the interaction energy $\omega_{12}$ are simultaneously obtained by solving Equations (16) and (17) by an iterative procedure.

\section{Results and Discussion}

The mole fraction of the complex $T l N a$ at eqiatomic composition and the model parameters $\omega_{12} / R T$ and $l n k+\omega_{13} / R T$ are first determine simultaneously by solving the Equations (16) and (17) with the help of observed values of activity coefficient [25] at equiatomic and zero compositions. Then the mole fractions of the complexes and the unassociated atoms are obtained from Equations (1) and (16a) with the aid of above determined parameters by iterative process. Figure 1 shows the compositional dependence of the mole fractions of the complex and the unassociated atoms. It is found that the maximum association occurs at 53.88 at pct. of $\mathrm{Tl}$ and at this composition about 50.85 mol pct. of the liquid alloy is associated.

The pairwise interaction energies and the equilibrium constant are calculated from Equations (5), (13) and (14) by the successive approximation to explain the observed values of free energy of mixing [25]. The best fit values so obtained for Tl-Na liquid alloy at $673 \mathrm{~K}$ are found to be

$$
\frac{\omega_{12}}{R T}=-1.68, \frac{\omega_{13}}{R T}=-2.31, \frac{\omega_{23}}{R T}=-0.986 \text { and } k=0.0347 .
$$

Figure 2 shows the well agreements between the theoretical values (Equation (5)) and the observed values of the free energy of mixing. Both the theoretical and the observed values of $\frac{G_{M}}{R T}$ are found to be minimum at $x_{T l}=0.5$ ( theoretical values of $\frac{G_{M}}{R T}=-2.1569$ and the observed value of $\frac{G_{M}}{R T}=$ $-2.2008 \pm 0.07472$ [25]). The observed values of excess free energy of mixing of Tl-Na liquid alloy at $673 \mathrm{~K}$ are moderately high indicating that this system is moderately interacting system. 


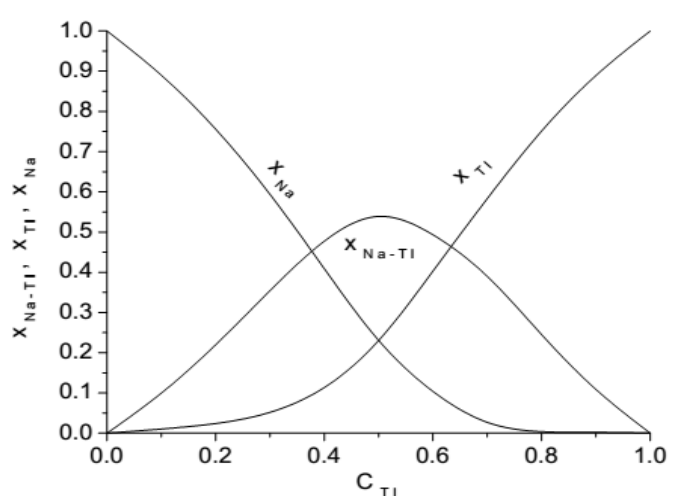

Fig. 1: Mole fractions of the complex (TlNa), unassociated atoms ( $\mathrm{Tl}$ and $\mathrm{Na}$ ) vs concentrations of In $\left(C_{T l}\right)$ of Tl-Na liquid alloy at $673 \mathrm{~K}$.

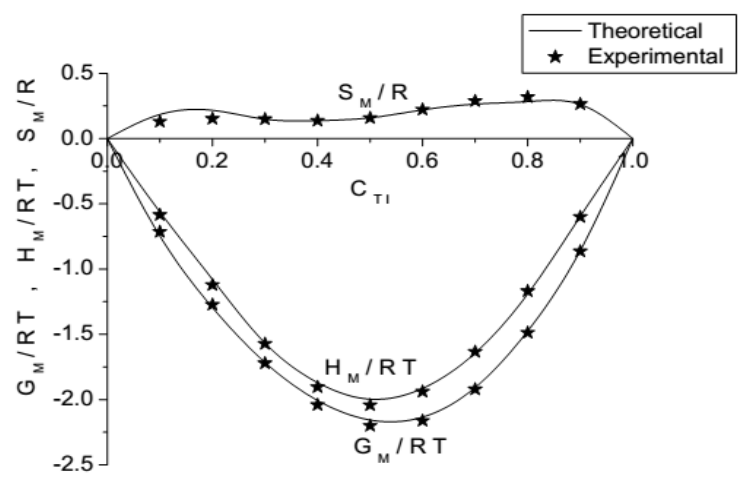

Fig. 2: Free energy of mixing $\left(G_{M} / R T\right)$, heat of mixing $\left(H_{M} / R T\right)$ and entropy of mixing $\left(S_{M} / R\right)$ vs concentrations of $\mathrm{Tl}\left(C_{T l}\right)$ of $\mathrm{Tl}-$ Na liquid alloy at $673 \mathrm{~K}$.

According to this model, all the interaction energy parameters are considered to be temperature dependent. However, if they are assumed independent of temperature then the expression for the enthalpy of mixing can be simply expressed as

$H_{M}=\frac{1}{1+\mu x_{A_{\mu} B}}\left[\left(x_{A} x_{B} \omega_{12}+x_{A} x_{A_{\mu} B} \omega_{13}+x_{B} x_{A_{\mu} B}\right)-x_{A_{\mu} B} R T^{2} \frac{d \ln k}{d T}\right]$

Then $H_{M}$ can be obtained with the knowledge of the interaction energy parameters between the unassociated species and to the complex and $R T^{2} \frac{d \operatorname{lnk}}{d T}$. The $H_{M}$ so obtained differ far from the observed values. This supports the importance of the temperature dependence of interaction energy parameters.

The temperature dependent model parameters are obtained using Equation (7) to explain the observed values of $H_{M}$ [25] by successive approximations. The best fit values of such parameters are found to be

$$
\frac{\partial \omega_{12}}{\partial T}=8.00, \quad \frac{\partial \omega_{13}}{\partial T}=13.67, \quad \frac{\partial \omega_{23}}{\partial T}=7.00 \text { and } R T^{2} \frac{d l n k}{d T}=20500 \pm 4500 .
$$

Both the theoretical (Equation (7)) and the observed values of $H_{M}$ are in well agreements (Figure 2). The minimum values of both of $H_{M}$ are found to be at $x_{T l}=0.5$ ( theoretical value of $\frac{H_{M}}{R T}=-1.9975$ and the observed value of $\left.\frac{H_{M}}{R T}=-2.0432 \pm 0.01868[25]\right)$.

The theoretical values of the entropy of mixing $\left(S_{M}\right)$ are obtained from the Equation (6b) with the knowledge of $G_{M}$ and $H_{M}$. Both the theoretical and the observed values of $S_{M}$ are in well agreements (Figure 2).

The compositional contributions of the heat of mixing of the species $\mathrm{x}_{\mathrm{A}}, \mathrm{x}_{\mathrm{B}}$ and $\mathrm{x}_{\mathrm{A}_{\mu} \mathrm{B}}$ and the heat associated with the formation of the complexes to the total enthalpy change are obtained from Equation (7). The contribution from the complex formation term is maximum at 47.46 at. pct. of $\mathrm{Tl}$ and the mixing term contributes the maximum at 67.79 at. pct. of $\mathrm{Tl}$ due to which the net enthalpy 
change shows absolute maximum at around 50.85 at. pct. of Tl. Figure 3 shows the compositional dependance of the heat of mixing, heat associated with the formation of the complexes and the total enthalpy change.

The theoretical values of the activities of the unassociated atoms of the liquid alloys are estimated using Equations (12). The model parameters obtained above for the present system must not only explain the thermodunamic properties but also must reproduce the activity. It is an important parameter having great influence on the thermodynamic as well as the structural properties of the liquid alloys. Any deviation from the ideality can be incorporated into the activity. Figure 4 shows the well greements between the theoretical and the observed values [25] of the activities.

The microscopic structural behaviour of the liquid alloys can be studied by estimating concentration fluctuation in long wavelength limit $\left(S_{C C}(0)\right)$. The theoretical and the experimental values of $S_{C C}(0)$ are respectively obtained from Equations (8) and (6d). Both the theoretical and the experimental values of $S_{C C}(0)$ are found to be less than the ideal values throughout the entire concentrations. The theoretical analysis thus suggest that there is unlike atoms pairing (ordering) in Tl-Na liquid alloy at $673 \mathrm{~K}$. Both the theoretical and the experimental values of $S_{C C}(0)$ are in reasonable agreements (figure 5).

The degree of local ordering of atoms in the liquid state can be studied by estimating the WarrenCowley short range order parameter $\left(\alpha_{1}\right)$. At the equiatomic composition, the values of $\alpha_{1}$ lies between -1 to +1 . If $\alpha_{1}=-1$, indicates the complete ordering of unlike atoms at nearest neighbours, if $\alpha_{1}=+1$, indicates the complete segregation or paring of like atoms in the nearest neighbours and if $\alpha_{1}=0$, indicates the complete randomness of the atoms in the liquid state. The compositional dependence of $\alpha_{1}$ is shown in Figure 6. It can be seen that the values of $\alpha_{1}$ are found to be negative at all compositions which further verifies the ordering nature of the liquid system .

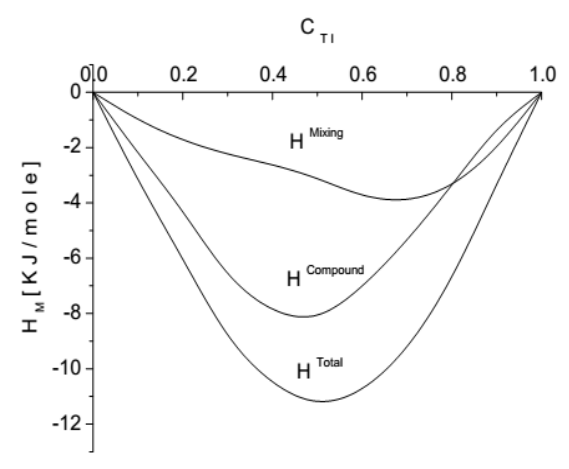

Fig. 3: Contributions of the heat of compound formation and heat of mixing to total enthalpy change vs concentrations of Tl $\left(C_{T l}\right)$ of Tl-Na liquid alloy at $673 \mathrm{~K}$.

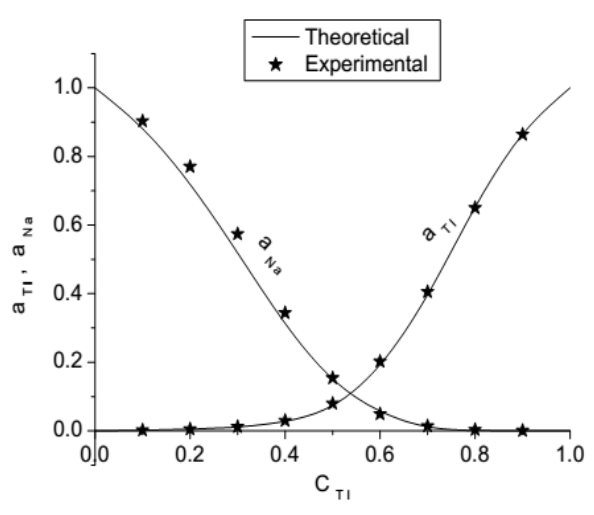

Fig. 4: Activities of $\mathrm{Tl}\left(a_{T l}\right)$ and $\mathrm{Na}$ $\left(a_{N a}\right)$ vs the concentration of $\mathrm{Tl}\left(C_{T l}\right)$ of Tl-Na liquid alloy at $673 \mathrm{~K}$. 
The mixing tendency of the liquid alloy can be studied at the microscopic level by computing the ratio of the mutual and intrinsic-diffusion coefficients $\left(D_{M} / D_{i d}\right)$. If $D_{M} / D_{i d}>1$, indicates the tendency of compound formation; if $D_{M} / D_{i d}<1$, indicates the separation of phase and $D_{M} / D_{i d}=1$, for the ideal mixing of the liquid alloy. The values of $D_{M} / D_{i d}$ are obtained from Equation (10) and found to be positive and greater than one in the entire compositions. This further strengthen the theoretical analysis of the presence of the chemical order in the liquid state of Tl-Na liquid alloy at $673 \mathrm{~K}$.

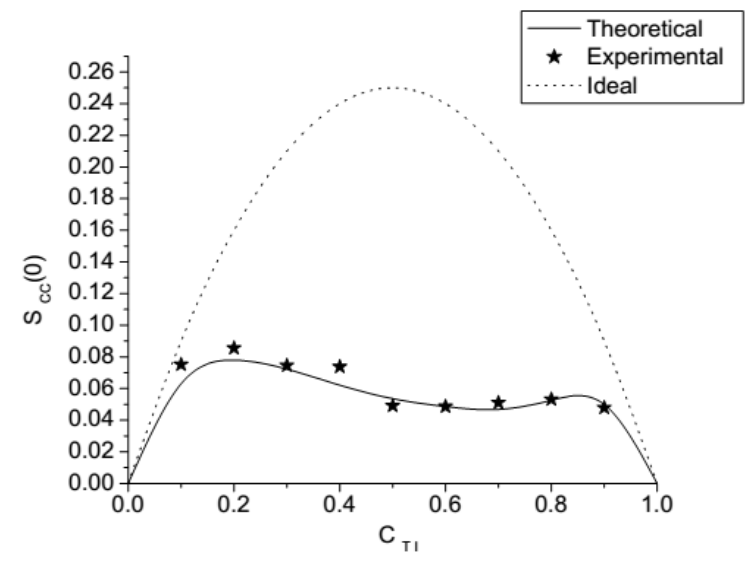

Fig. 5: Concentration fluctuation in long wavelength limit $\left(S_{C C}(0)\right)$ vs the concentration of $\mathrm{Tl}\left(C_{T l}\right)$ of Tl-Na liquid alloy at $673 \mathrm{~K}$.

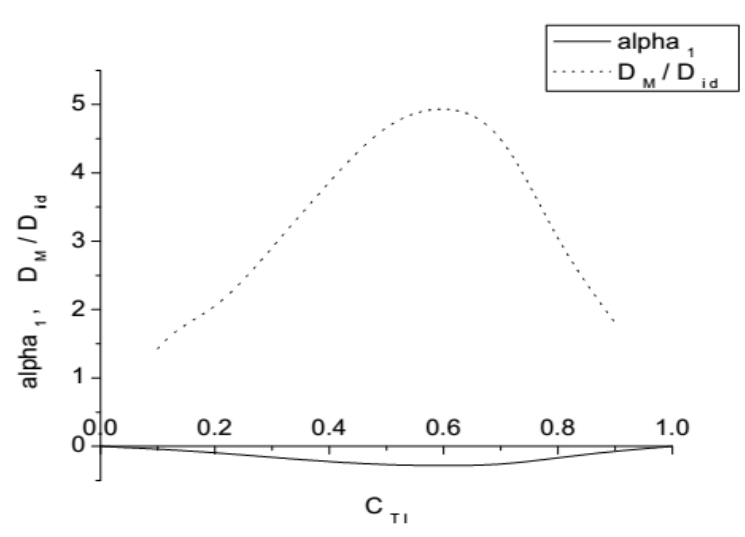

Fig. 6: Short-range order parameter $\left(\alpha_{1}\right)$ and the ratio of mutual and intrinsic coefficients $\left(D_{M} / D_{i d}\right)$ vs the concentration of Tl $\left(C_{T l}\right)$ of Tl-Na liquid alloy at $673 \mathrm{~K}$.

\section{Conclusions}

The theoretical analysis of the Tl-Na liquid alloy at $673 \mathrm{~K}$ indicates that the interaction energy parameters are temperature dependent and there is tendency of chemical ordering in this system. The system under consideration is found to be moderately interacting.

\section{References}

[1] F. Sommer, J. Non-Cryst. Solids, 117 (1990) 505. http://dx.doi.org/10.1016/0022-3093(90)90580-F

[2] K. Hoshino, J. Phys. F: Met. Phys., 13 (1983) 1981. http://dx.doi.org/10.1016/0022-3093(90)90580-F

[3] R.N. Singh, F. Sommer, J. Phys. Condens. Matter., 4 (1992) 5345. http://dx.doi.org/10.1088/0953-8984/4/24/004 
[4] D. Adhilari, Phase Transitions, 84 (2010) 308. http://dx.doi.org/10.1080/01411594.2010.536088

[5] W.H. Young, Rep. Prog. Phys., 55 (1992) 1769. http://dx.doi.org/10.1088/0034-4885/55/10/003

[6] D. Adhikari, B.P. Singh, I.S. Jha, Phys. Chem. Liq., 48 (2010) 787. http://dx.doi.org/10.1080/00319101003699008

[7] H. Ruppersberg, H. Reiter, J. Phys. F: Met. Phys., 12 (1982) 1311. http://dx.doi.org/10.1088/0305-4608/12/7/005

[8] P.J. Flory, J.Chem. Phys., 10 (1942) 51. http://dx.doi.org/10.1063/1.1723621

[9] A.B. Bhatia, R.N. Singh, Phys. Chem. Liq., 13 (1984) 177. http://dx.doi.org/10.1080/00319108408080778

[10] B.P. Albas, W. Van der Lugt, J. Dijkstra, W. Geertsma, C. VanDijik, J. Phys. F: Metal Phys. 13 (1983) 2465. http://dx.doi.org/10.1088/0305-4608/13/12/007

[11] R.P. Godbole, S.A. Jha, M. Milanarun, A.K. Mishra, J. Alloys Compd., 363 (2004) 182.

http://dx.doi.org/10.1016/S0925-8388(03)00326-8

[12] A.B. Bhatia, W.H. Hagrove, Phys. Rev., 10 (1974) 3186. http://dx.doi.org/10.1103/PhysRevB.10.3186

[13] La. Dou, J. Metals. In., (1997) 429.

[14] C. Wang, Y. Chen, J. Liu, J. Wang, X. Li, Y. Zhang, Ecotoxicol Environ Saf., 90 (2013) 76-81. http://dx.doi.org/10.1016/j.ecoenv.2012.12.014

[15] A. Turner, O. Furniss, Mar Pollut Bull., 64 (2012) 2720-2724. http://dx.doi.org/10.1016/j.marpolbul.2012.09.023

[16] F. Habashi, Alloys: Preparation, Properties and Applications, John Wiley and Sons. (2008).

[17] D. Adhikari, S.K. Yadav, L.N. Jha, Journal of the Chinese Advanced Materials Society, 2(3) (2014) 149-158.

http://dx.doi.org/10.1080/22243682.2014.928603 
[18] D. Adhikari, I.S. Jha, J. Kumar, B.P Singh, J. Mol. Struct., 985 (2011) 91. http://dx.doi.org/10.1016/j.molstruc.2010.10.026

[19] S. Lele, P. Ramchandrarao, Metall. Trans., 12B (1981) 659. http://dx.doi.org/10.1007/BF02654134

[20] D. Adhikari, B.P. Singh, I.S. Jha, B.K. Singh, J. Mol. Liq., 156 (2010) 115. http://dx.doi.org/10.1016/j.molliq.2010.05.020

[21] A.S. Jordan, Metall. Trans., 1 (1970) 239.

[22] D. Adhikari, B.P. Singh, I.S. Jha, J. Non-Cryst. Solids., 358 (2012) 1362. http://dx.doi.org/10.1016/j.jnoncrysol.2012.03.008

[23] I. Prigonine, R. Defay, Chem. Thermodynamics, Longmans Green and Co.,London (1974) 257.

[24] K. Osamura, B. Predel, Trans. Jpn. Inst. Met., 118 (1977) 765-75. http://dx.doi.org/10.2320/matertrans1960.18.765

[25] R. Hultgren, P.D. Desai, D.T. Hawkins, M. Gleiser, K.K. Kelley, Selected Values of the Thermodynamic Properties of Binary Alloys, ASM, Metal Park1 (973).

[26] T.S. Yih, J.C. Thompson, J. Phys. F. Met. Phys., 12 (1982) 1625. http://dx.doi.org/10.1088/0305-4608/12/8/009

[27] R.N. Singh, Can. J. Phys., 65 (1987) 309. http://dx.doi.org/10.1139/p87-038

[28] R. Novakovic, J. Non-Cryst. Solids, 356 (2010) 1593. http://dx.doi.org/10.1016/j.jnoncrysol.2010.05.055

[29] R. Singh, F. Sommer, Phys. Chem. Liq., 36 (1998) 17. http://dx.doi.org/10.1080/00319109808035917

[30] L. Darken, R. Gurry, Physical Chemistry of Metals, New York: McGraw Hill (1953). 